SCHOLARONE ${ }^{m}$

Manuscripts
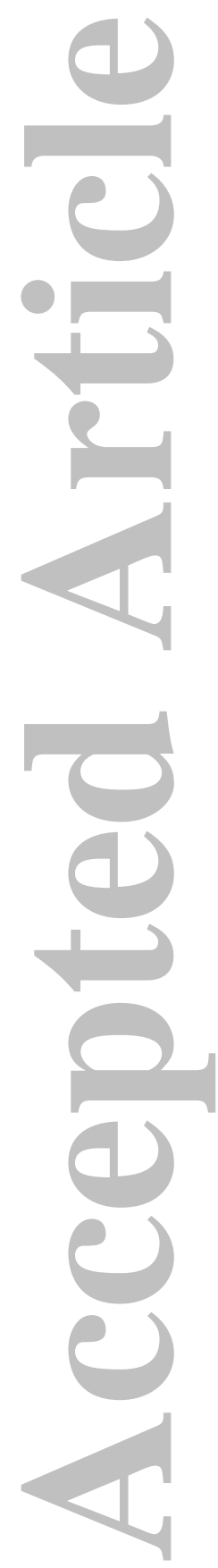

This is the author manuscript accepted for publication and has undergone full peer review but has not been through the copyediting, typesetting, pagination and proofreading process, which may lead to differences between this version and the Version record. Please cite this article as doi:10.1111/ cid.12541.

This article is protected by copyright. All rights reserved. 


\section{A comparative study of Encode protocol versus conventional protocol for restoring single implants: one- year prospective randomized controlled clinical trial}

Abbreviated title: Encode protocol for restoring single implants

Jaafar Abduo, BDS, DClinDent, PhD, MRACDS (Pros)

Senior Lecturer in Prosthodontics, Convenor of Postgraduate Diploma in Clinical Dentistry (Implants)

Melbourne Dental School, Melbourne University, 720 Swanston Street, Melbourne, Victoria 3010

Lakshmi Gade, BDS, PgradDipCD

Former Postgraduate Student

Melbourne Dental School, Melbourne University, 720 Swanston Street, Melbourne, Victoria 3010

Harjot Gill, BDS, PgradDipCD

Former Postgraduate Student

Melbourne Dental School, Melbourne University, 720 Swanston Street, Melbourne, Victoria 3010

Roy Judge, BDS, LDS RCS, MDSc, PhD

Associate Professor in Prosthodontics

Melbourne Dental School, Melbourne University, 720 Swanston Street, Melbourne, Victoria 3010

Ivan Darby, BDS, PhD, FRACDS(Perio), DGDP RCSEng, FADI

Head of Periodontics

Melbourne Dental School, Melbourne University, 720 Swanston Street, Melbourne, Victoria 3010

Corresponding Author: Dr Jaafar Abduo, Melbourne Dental School, Melbourne University, 720 Swanston Street, Melbourne, Victoria 3010

Phone: +61390358998

Fax: $\quad+61393411599$

Email address: jaafar.abduo@unimelb.edu.au

The authors have no conflict of interest.

Key words: clinical study, CAD/CAM, titanium, abutment 


\section{Authors' contribution}

Jaafar Abduo

- Study design

- Data analysis and interpretation

- Manuscript outline

- Re-writing and critical revision of the article

- Approval of article

- Agreement to be accountable for all aspects of the work in ensuring that questions related to the accuracy or integrity of any parts of the work are appropriately investigated and resolved

\section{Lakshmi Gade}

- Clinical data collection

- Data analysis and interpretation

- Writing the $1^{\text {st }}$ manuscript draft (introduction and discussion)

- Approval of article Agreement to be accountable for all aspects of the work in ensuring that questions related to the accuracy or integrity of any parts of the work are appropriately investigated and resolved

\section{Harjot Gill}

- Clinical data collection

- Data analysis and statistics

- Writing the $1^{\text {st }}$ manuscript draft (methodology and results)

- Approval of article

- Agreement to be accountable for all aspects of the work in ensuring that questions related to the accuracy or integrity of any parts of the work are appropriately investigated and resolved

\section{Roy Judge}

- Study design

- Restorative data interpretation

- Critical revision of the article

- Approval of article

- Agreement to be accountable for all aspects of the work in ensuring that questions related to the accuracy or integrity of any parts of the work are appropriately investigated and resolved

\section{Ivan Darby}

- Study design

- Periodontal data interpretation

- Critical revision of the article

- Approval of article

- Agreement to be accountable for all aspects of the work in ensuring that questions related to the accuracy or integrity of any parts of the work are appropriately investigated and resolved 


\section{A comparative study of Encode protocol versus}

\section{conventional protocol for restoring single implants: one-}

\section{year prospective randomized controlled clinical trial}

\section{Abstract}

Background: The Encode protocol (Biomet 3i, Palm Beach Gardens, Fla, USA) has been proposed as a simplified implant restoration protocol.

Purpose: To compare the 1-year clinical outcome of the Encode and conventional protocols for restoring single implants.

Materials and methods: Forty seven implants were inserted in 44 patients. After randomizing the implants, 24 implants were allocated to the Encode protocol and 23 implants were allocated to the conventional protocol. After 1 year, changes in aesthetics, patient satisfaction, proximal contacts quality, occlusal contacts quality, marginal bone level $(\mathrm{MBL})$, and probing pocket depth (PPD) were evaluated. Further, the prosthesis cleansability, mucosal health, bleeding on probing (BoP), metallic discoloration and all forms complications were recorded.

Results: Forty patients with 41 implants (22 Encode and 19 conventional) were recalled. One conventional crown failed due to excessive looseness. Aesthetics, patient satisfaction and prosthesis cleansability were favourable for the 2 protocols. One Encode crown (4.5\%) and 6 conventional crowns (33.3\%) had slight mucosal redness. BoP was present around 8 Encode crowns (36.4\%) and 8 conventional crowns (45.4\%). Only 2 conventional crowns showed metallic discoloration of the mucosa. The 2 protocols had similar PPD alteration $($ Encode $=0.04 \mathrm{~mm}$, conventional $=0.13 \mathrm{~mm})$, and $\mathrm{MBL}$ loss $($ Encode $=0.71 \mathrm{~mm}$, 
conventional $=0.78 \mathrm{~mm}$ ). Similar proximal contacts and occlusal contacts were observed for the 2 protocols.

Conclusions: After 1 year, the Encode protocol for restoring single implants appears to be comparable to the conventional protocol from the biological, prosthetic and aesthetic perspectives.

\section{KEYWORDS}

(

\section{clinical study, computer-assisted, titanium, implant}

\section{INTRODUCTION}

Oral Implant prostheses are favourable treatment modality for partially or completely edentulous patients in contemporary dental practice. Since the introduction of oral implants, there have been several attempts to simplify the implant treatment and enhance the clinical outcome. This had involved modifying the impression technique and utilizing new manufacturing technologies such as computer-aided design and computer-aided manufacturing (CAD/CAM). Traditionally, implant impression is taken via open or close tray technique with impression coping and elastomeric impression material. Such protocol mandates the removal of the healing abutment before seating the impression coping on the implant. During this procedure multiple components may require fitting and handling, which can cause discomfort and complications such as soft tissue irritation. ${ }^{1,2}$

Encode protocol (Biomet 3i, Palm Beach Gardens, Fla, USA) has been proposed as an alternative approach to conventional impression. The Encode protocol involves fitting a two-piece coded healing abutment at the time of implant placement. The uniqueness of the two-piece healing abutment comes from the surface codes that transfer information about implant connection, diameter, vertical placement and hex orientation. ${ }^{3-5}$ The impression of 
the Encode protocol is taken by whole arch close tray technique without removing the healing abutment. After pouring the impression, the stone cast is transferred to a centralized production centre (Biomet $3 \mathrm{i}$ scanning/milling centre, Fla, USA) for scanning. The information held in the codes of the healing abutment is used to virtually determine the implant location and orientation. This is followed by designing and fabricating a customized titanium abutment by CAD/CAM. In addition, the virtual information is used to dictate implant replica placement within the stone cast. ${ }^{3-5}$ The titanium abutment and the modified stone cast are returned to the manufacturing dental technician for the fabrication of the definitive implant crown.

The speculated advantages of the Encode protocol are simplicity, reduction of impression procedure duration, ${ }^{6,7}$ enhanced patient comfort and reduction of peri-implant soft tissue irritation. ${ }^{3,8-10}$ Further, it is deemed easier to take implant impressions by the Encode protocol for posterior region where access is limited. Since the Encode protocol involves production of a CAD/CAM abutment, the abutment will be customized which ensures ideal contour incorporation and crown margin placement. ${ }^{6}$

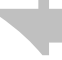

Currently, there are few clinical reports describing the merits of the Encode protocol. ${ }^{3,6,7}$ However, long-term clinical comparison against conventional protocol in restoring implants is still lacking. Therefore, the aim of this prospective randomized controlled clinical trial is to compare the 1-year outcome of the Encode protocol to the conventional protocol for restoring single implants. This study is a follow-up study of a recently published report on the baseline outcome of the Encode and the conventional protocols. ${ }^{6}$ The hypothesis is the two protocols will yield similar clinical outcome. 


\section{MATERIALS AND METHODS}

\subsection{Study design and power analysis}

A total of 47 3i Biomet implants (OSSEOTITE Implant, Biomet 3i, Palm Beach Gardens, Fla, USA) were inserted in 44 patients. Three patients received 2 nonadjacent implants. The implants were randomly allocated to Encode group (24 implants) or conventional group (23 implants) by sequential allocation of identification numbers generated by computer randomisation. Enrolment of participants, randomisation of implants and assigning of participants to interventions were conducted by a dedicated research administrator. The number of implants was based on earlier studies ${ }^{11,12}$ and was confirmed by power calculation using G*Power software (version 3.1.9.2; University of Düsseldorf, Düsseldorf, Germany). The assumptions of $80 \%$ statistical power and $5 \%$ significance level revealed that at least 21 implants are needed in each group. Double blinding was not feasible since the restoring clinicians needed to be aware of the selected treatment protocol. All clinical steps were provided at the Royal Dental Hospital of Melbourne (RDHM) and approved by the University of Melbourne Human Research Ethics Committee (1034995.2). The participants were given plain-language statement explaining the study before obtaining informed written consent. The participants had the option to withdraw from the study at any period. The CONSORT guidelines for clinical studies were followed in this study ${ }^{13}$ (Figure 1). The treatment was completed by a group of postgraduate students. Patients were selected if they were at least 18 years old, had a minimum of 20 teeth, required single implant restoration(s) and were physically fit and able to tolerate implant surgical and restorative procedures. Patients with uncontrolled diabetes, head and neck radiation, severe parafunction, metabolic bone diseases, pregnancy, active caries or periodontal disease, major bone grafting or smoking habit were excluded. 


\subsection{Surgical and restorative protocols}

One- or two-stage surgical protocol was followed to insert the implants. All the implants received Encode healing abutments with approximately $2 \mathrm{~mm}$ height above the soft tissue at the time of implant surgery or second stage surgery. After the surgery, a periapical radiograph was taken to ensure accurate seating of the Encode abutment. The sutures were removed after 2 weeks and implant osseointegration was confirmed after 3 months by a forward $20 \mathrm{Ncm}$ torque test through the healing abutment.

All the impressions were taken by a combination of light and heavy body polyvinyl siloxane impression materials (Aquasil, Dentsply Caulk, Milford, DE, USA). For the Encode group, the impression was taken by a custom close tray without the removal of the healing abutment. For the conventional group, the impression was taken by a custom open tray and implant level impression coping. All impressions were sent to a single commercial dental laboratory that was familiar with the two impression protocols. One technician fabricated all the definitive restorations.

The implants of both groups were restored with porcelain fused to metal (PFM) crowns fitted on abutments and retained by cross-pin (lateral prosthetic screw) to ensure retrievability. $^{6,14}$ For the Encode protocol, implant casts were sent to Biomet $3 \mathrm{i}$ scanning/milling centre in Florida for scanning, CAD/CAM abutments fabrication, and an implant replica insertion in the cast. The milled abutments and the modified casts were sent back to the dental laboratory for final crown fabrication. After pouring the conventional impressions, stock titanium abutments were attached on the implant replicas. Stock abutments were used for the conventional group to ensure similarity in components number, retention mechanism and degree of fit on the implant with the Encode group. In most conventional group cases, the stock abutments required manual customization to improve the crown design. Following the clinical insertion of the abutment and the crown, a periapical radiograph was taken to confirm accurate fit of the prosthesis and to record the baseline 
marginal bone level $(\mathrm{MBL})$. In situations where major adjustments of the crown were indicated, it was sent back to the laboratory. If no or minimal adjustments were required, the abutment screw was tightened to the manufacturer's recommended value and the cross-pin was hand-tightened.

\subsection{Clinical evaluation and data collection}

To standardize the data collection, questionnaires were completed by the operator at every clinical visit. Further, the inter-examiner reliability was enhanced by calibrating the operators on the data collection procedures and questionnaires completion. The data collection procedures were supervised by an experienced clinician who is familiar with the project. At the time of insertion, baseline proximal contacts and occlusal contacts were evaluated. The proximal contacts were evaluated with dental floss (Colgate Dental Ribbon, New York, USA) and foil shim stock (Coltene/Whaledent, Langenau, Germany). The criterion for an ideal contact was that it provided some resistance to interproximal floss or shim stock insertion.

An open contact was reported if there was no resistance against floss or shim stock, while tight contact prevented the passage of floss through the proximal contact point. For the proximal contact analysis, the outcomes of the mesial and distal contacts were combined. An evaluation of occlusion was executed with shim stock, and the planned occlusal contact should hold the shim stock when the patient clenched. If the shim stock was held after simple closure, the occlusal contact was reported as heavy and received mild chairside adjustment. If the shim stock passed without resistance after clenching, an open contact was

reported. If the crown is returned to the manufacturing technician, the adjustment is considered major. Post-insertion review was scheduled after 1-4 weeks (baseline) to evaluate aesthetics, patient satisfaction and probing pocket depth (PPD). The crowns aesthetics were assessed subjectively by the patient and the clinician as acceptable or unacceptable (shape, shade or combination of both). Patient satisfaction with the prosthesis 
was reported on a scale of 1-10 (10 being the highest). With the aid of Williams probe (HuFriedy Mfg. Co., LLC, Chicago, IL, USA), the PPD was measured in 6 sites around each implant crown: mesiobuccal, midbuccal, distobuccal, mesiolingual, midlingual and distolingual.

After 1 year, the patients were recalled to review the aesthetics, satisfaction, biological and prosthetic outcomes. A periapical radiograph was taken for each implant. The aesthetics and patient satisfaction were recorded similar to baseline. The evaluated biological variables were the cleansability of the prosthesis, mucosal health (signs of inflammation), bleeding on probing (BoP) (present or absent), metallic discoloration (present or absent), PPD, and MBL. The PPD and MBL were compared against the baseline data. The base line and the 1-year radiographs were digitized, and the distance between the implant platform and the most coronal crestal bone was measured. The distance between 2 implant threads was used for image calibration and exact determination of the bone level. As per baseline evaluation, the proximal and occlusal contacts were evaluated. In addition, any form of biological and prosthetic complications was documented and used to calculate the survival of the implants and the prostheses.

\subsection{Statistics}

The data from written questionnaires were imported into SPSS statistics software (SPSS for Windows, version 23 , SPSS Inc., Chicago, IL, USA). The implant was the statistical unit of analysis. The data was analysed with descriptive and analytical statistics. The Shapiro-Wilk test was used to establish that the distribution of the variables tested that was not normal. The Mann-Whitney U-test was used to compare continuous variables (patient satisfaction, PPD and MBL changes). Categorical variables (hygiene, mucosal health, BoP, metallic discoloration, proximal contacts and occlusion) of the two protocols were evaluated by the chi-square test. The level of significance was set at 0.05 . 


\section{RESULTS}

One early implant failure happened in the conventional group before restoration and was subsequently excluded from the study. After 1 year, 3 patients with 5 implants were unable to attend the review visit. Therefore, 40 patients with 41 implants presented at the recall visit $(87.0 \%$ recall rate). Of all the restorations, 22 were Encode and 19 were conventional restorations. All the implants maintained osseointegration. Therefore, the 1-year survival rate of the implants was $100 \%$ for the 2 protocols. One conventional crown failed and was subsequently excluded from further analysis in the study. Therefore, the 1-year prosthesis survival rate of the 2 protocols is $97.6 \%$.

\subsection{Patient satisfaction and aesthetics}

At baseline, the mean patient satisfaction was $9(S D=1.4)$ for the Encode group and 9.5 $(S D=1.5)$ for the conventional group. After 1 year, the mean patient satisfaction was 9 for the two groups. The patient satisfaction had improved for 10 Encode $(45.5 \%)$ and 3 conventional (16.7\%). For 4 Encode $(18.2 \%)$ and 6 conventional $(33.3 \%)$ restorations, the patient satisfaction was reduced. For the remaining implants, 8 Encode (36.4\%) and 9 conventional $(50.0 \%)$, no changes in satisfaction were observed. There was no significant difference for patient satisfaction between Encode and conventional restorations $(P=.15)$. In relation to restoration aesthetics, all the restorations were rated as acceptable by the patient at baseline and at 1-year review.

\subsection{Biological factors}

For the 2 groups, all the crowns were cleansable and the patients demonstrated good standards of oral hygiene around the implant crowns. Out of 22 Encode implants, 21 (95.5\%) had healthy mucosa and one showed slight redness (4.5\%). Twelve conventional crowns $(66.7 \%)$ had healthy mucosa and $6(33.3 \%)$ had minor mucosal redness. There was no 
significant difference between the 2 groups $(P=.10)$. In reference to BoP, 14 Encode (63.6\%) had no BoP and 8 Encode $(36.4 \%)$ had BoP. For the conventional implants, 10 $(55.6 \%)$ had no BoP and 8 (45.4) had BoP. The two groups were similar in relation BoP $(P=$ .89). Metallic discoloration was present in only two conventional implants (11.1\%). None of the Encode restorations showed signs of metallic discoloration. However, the metallic discoloration of soft tissue was similar in both techniques $(P=.33)$.

For the two groups, the PPD had minimally changed over the 1-year period $(0.08$ $\mathrm{mm}, 95 \% \mathrm{Cl}:-0.36$ to $0.52 \mathrm{~mm} ; P=.70)$. This relationship was observed for the Encode group $(0.04 \mathrm{~mm}, 95 \% \mathrm{Cl}:-0.58$ to $0.66 \mathrm{~mm} ; P=.85)$ and the conventional group $(0.13 \mathrm{~mm}$, $95 \% \mathrm{Cl}:-0.47$ to $0.73 \mathrm{~mm} ; P=.51)$. Between the two groups, there was minimal differences in the changes of the PPD $(P=.65)$. Table 1 outlines the average PPD of each group at baseline and after 1 year, and the average difference between the two evaluation periods.

The average reduction of $\mathrm{MBL}$ for all the implants was $0.74 \mathrm{~mm}(95 \% \mathrm{Cl}: 0.33$ to

$1.15 \mathrm{~mm})$. The reduction of MBL was $0.71 \mathrm{~mm}(95 \% \mathrm{Cl}: 0.15$ to $1.27 \mathrm{~mm})$ for the Encode group, and $0.78 \mathrm{~mm}(95 \% \mathrm{Cl}: 0.16$ to $1.40 \mathrm{~mm}$ ) for the conventional group. The difference between the two groups was negligible $(P=.97)$.

\subsection{Prosthetic factors}

Table 2 summarizes the proximal contacts and occlusal contacts quality at baseline and after 1 year. A total of 77 mesial and distal proximal contact points were included in the analysis. After 1 year of service, Encode crowns had 8 open contacts (18.2\%) and conventional crowns had 5 open contacts (13.9\%). There were 34 ideal contacts in Encode group (77.3\%) and 30 in conventional group (83.3\%). Eight proximal contacts of the Encode crowns $(18.2 \%)$ and 5 proximal contacts of the conventional crowns (13.9\%) were rated as 
open. None of the crowns had tight proximal contact. There was no significant difference in proximal contacts quality between the Encode and conventional techniques $(P=.93)$.

In relation to occlusion, 19 Encode (86.4\%) and 17 conventional $(94.4 \%)$ crowns required no adjustment. Two Encode crowns (9.1\%) and one conventional crown (5.6\%) needed mild chair side adjustment. One Encode crown was out of occlusion (4.5\%). There was no significant difference between the 2 techniques $(P=.59)$.

All the abutments were intact and abutment screw loosening was not observed. For the Encode group, 1 crown became mobile due to cross-pin loosening $(4.5 \%$ screw loosening rate). This was managed by gasket application of temporary cementation material and retightening of the cross-pin. Therefore, the Encode group had a survival of $100 \%$. The conventional group had 2 loose crowns and they were related to the cross-pin loosening $(10.5 \%$ screw loosening rate). One of the loose crowns was managed by gasket addition and retightening of the cross-pin. The other crown did not improve by the gasket addition, and the abutment design lacked the retentive features. As a result, this crown had to be remade. In addition, the conventional group had 2 crowns with ceramic chipping $(11.1 \%$ ceramic chipping rate). One of the crowns that suffered from ceramic chipping had to be sent to laboratory for ceramic addition. The chipping on the other crown was minor and was managed by chairside polishing. The survival rate of the conventional group was $94.7 \%$.

\section{DISCUSSION}

The results of this study indicate that the Encode and the conventional protocols are similar in terms of patient satisfaction, aesthetics, biological variable and prosthetic variables at one year. The Encode can produce a similar clinical outcome to the conventional restorative implant treatment. The outcome of this study is in accordance with the 2-year preliminary report by Vafiadis that showed favourable clinical outcome of the Encode protocol. ${ }^{7}$ 
The two protocols were similar in relation to cleansability, mucosal health, BoP, metallic discoloration, PPD and MBL. In general, the biological outcome of this study is comparable to previously published work on single implant restorations. For example, the PPD for the two groups fit within the range $(2.5-3.3 \mathrm{~mm})$ reported by earlier 1-year follow-up studies. $^{11,12,15,16}$ The average MBL reduction for all the implants was $0.74 \mathrm{~mm}$, which fits within the successful osseointegration criteria. ${ }^{17,18}$ In addition, it is comparable to previous observations after 1 year of evaluation. ${ }^{15,16,19}$ Similarly, the observed incidence of BoP around the implants was comparable with earlier research (about 50\%). ${ }^{12,19}$

Slightly more mucosal inflammation was observed around the conventionally fabricated restorations than around the Encode restorations. This could be due to the use of stock abutments in the conventional technique. For the Encode restoration, the peri-implant tissues contour is established by the CAD/CAM abutment. In addition, the customization of the CAD/CAM abutment will ensure placement of the crown margin near the contour of the soft tissue margins. ${ }^{20}$ Thus, the crown-abutment interface will still be accessible to routine cleaning by the patient which may contribute to the improved health of the soft tissue. For the conventional group, due to the use of stock abutments that are narrower than customizable abutment, the soft tissue contour has to be modified by the final crown. In order to achieve this, the crown margin may be well-below the soft tissue margin ${ }^{12}$ which will hinder routine cleaning of the crown-abutment interface. Although the crowns were retained by cross-pin, leakage and plaque accumulation may still occur and lead to soft tissue redness and inflammation. Such observation corroborates the outcome of earlier studies that reported advantages of the CAD/CAM abutment in maintaining soft tissue contour. ${ }^{21,22}$ Nevertheless, it is important to note that the biological differences between the two protocols were very minor and may not be of clinical significance or lead to biological and aesthetic failure. An additional advantage of the Encode protocol is the minimal intervention around the peri-implant tissue which may contribute to less irritation and inflammation. ${ }^{2}$ It is difficult to postulate that the minimal intervention is responsible for less inflammation, since the 
conventional impression technique has been the standard procedure for implant restoration for several decades.

The abutments of the two protocols survived the duration of the study without abutment fracture or screw loosening. Such an outcome is similar to other earlier studies that reported no stock or CAD/CAM abutments complications in the first few years of service. ${ }^{11,12,23-25}$ In addition, the survival of the restorations of the two protocols $(97.6 \%)$ is within the reported range by previous studies. ${ }^{12,25}$ In this study, the rate of mechanical complications was $14.8 \%$, of which $5 \%$ were for ceramic chipping and $9.8 \%$ for cross-pin loosening. Such an observation is in accordance with the most frequently reported mechanical complications. The most common types of mechanical complications are abutment screw loosening and veneering ceramic chipping. ${ }^{24,26,27}$ The ceramic complication rate has been frequently reported by earlier literature and appears to be a common problem that has been attributed to the lack of periodontal ligament around implants. For example, Sailer et al reported minor ceramic chipping rate of $16.7 \%$ of PFM crowns on milled titanium abutments after 1 -year of service. ${ }^{12}$ In relation to screw loosening, Jung et al found in a systematic review that the cumulative incidence of screw loosening of $12.7 \% .{ }^{27}$ In this study, the screw loosening occurred for the cross-pin and may be attributed to the minimal torque applied to tighten the screw, short screw length and horizontal orientation of the cross-pin in relation to the applied occlusal forces. The higher rate of cross-pin loosening was reported by other clinical studies. In a retrospective study, Krennmair et al found after 7 years of service, 3 out of 31 horizontal screws suffered from multiple loosening events, as opposed to 1 abutment screw loosening. ${ }^{28}$ More recently, in 5-year retrospective study, Lee et al reported noticeable cross-pin complications. Out of 73 implant crowns, 15 had mechanical complications of which $60 \%$ were related to cross-pin loosening. ${ }^{29}$ However, this is a minor issue and may require addition of gasket which is a simple clinical procedure. ${ }^{30}$

Despite the statistical similarity in survival rates of crowns fabricated on stock and Encode abutments at 1-year of clinical observation, the Encode protocol appears to be more 
advantageous than conventional protocol by reducing the rate of complications (ceramic chipping and screw loosening). This advantage can be related to the Encode abutment being fabricated via CAD/CAM processing which ensures abutment customization. Customized abutments are designed to follow anatomical contour which control the thickness of the final crown. ${ }^{7}$ As a result, it is likely that the final ceramic veneer has controlled thickness and is fully supported by the crown coping, which reduces the incidence and severity of veneering ceramic chipping. ${ }^{20}$ On the contrary, stock abutments lack the anatomical contour and the bulk of the restoration is achieved by the final crown. ${ }^{6}$ As a result, the final ceramic veneer may exhibit inconsistency in thickness and may be prone to more mechanical complications. In addition, due to the anatomical contour of CAD/CAM abutment, it will have more resistance and retention feature that will stabilize the crown and reduce the burden on the cross-pin. However, the cylindrical morphology of the stock abutment mean the crown is more prone for rotation and subsequent greater rate of the cross-pin loosening.

Overall, the two protocols had comparable quality of occlusion and proximal contacts. In comparison to baseline, the proximal contacts of the two protocols had no tight contacts, which can be attributed to the settling of the dentition around the implant over time. This was found to reduce the intensity of proximal contacts between implant crowns and adjacent natural teeth. ${ }^{31}$ Nevertheless, regardless of the restoration protocol the quality of occlusion and proximal contacts will be similar and require similar amount of clinical adjustment at baseline and after 1 year.

While this study is the first randomized controlled trial on the clinical outcome of the Encode protocol for single implant restoration in comparison to conventional protocol, it suffers from few limitations. For example, the trial included relatively few implants in the two groups. Further, the short follow-up period cannot detect the long-term implication of each protocol. The clinical treatment was provided in a university setting by group of postgraduate students which may cause inevitable variation. Since the inter- and intra-examiner tests were 
not conducted, further loss of study power is anticipated. The variation from multiple operators was potentially controlled by calibration and following questionnaire template. An additional source of limitation is the lack of blinding of examining clinicians. Since the treatment information were documented in each patient's file, it was not feasible to blind the clinicians from the groups patients were allocated to. While the study indicates the overall similarity in the clinical outcome of the Encode protocol to the conventional protocol for single implant restoration, more research is needed to confirm the long-term performance and the outcome for multi-unit restorations.

In conclusion, within the limitations of this study, it can be concluded that the Encode and conventional protocols are comparable from the biological, prosthetic and aesthetic perspectives after 1 year of service. Some advantages of the Encode protocol were observed and they can be attributed to CAD/CAM manufacturing of titanium abutments.

\section{ACKNOWLEDGEMENTS}

This study has been supported by Biomet $3 \mathrm{i}$. The authors acknowledge the assistance provided by the staff of the Royal Dental Hospital of Melbourne and Studio Dental Laboratory. The authors would also like to thank Ms Karen Escobar and Mr Geoff Adams for their generous contribution in data analysis. The authors declare no conflict of interest.

\section{CONFLICT OF INTEREST}

The authors declare no conflict of interest. 


\section{REFERENCES}

[1] Lee H, So JS, Hochstedler JL, Ercoli C. The accuracy of implant impressions: a systematic review. J Prosthet Dent. 2008;100(4):285-291.

[2] Abrahamsson I, Berglundh T, Lindhe J. The mucosal barrier following abutment dis/reconnection. An experimental study in dogs. J Clin Periodontol. 1997;24(8):568572.

[3] Grossmann Y, Pasciuta M, Finger IM. A novel technique using a coded healing abutment for the fabrication of a CAD/CAM titanium abutment for an implant-supported restoration. J Prosthet Dent. 2006;95(3):258-261.

[4] Telleman G, Raghoebar GM, Vissink A, Meijer HJ. The use of a coded healing abutment as an impression coping to design and mill an individualized anatomic abutment: a clinical report. J Prosthet Dent. 2011;105(5):282-285.

[5] Nayyar N, Yilmaz B, McGlumphy E. Using digitally coded healing abutments and an intraoral scanner to fabricate implant-supported, cement-retained restorations. $J$ Prosthet Dent. 2013;109(4):210-215.

[6] Abduo J, Chen C, Le Breton E, et al. The Effect of Coded Healing Abutments on Treatment Duration and Clinical Outcome: A Randomized Controlled Clinical Trial Comparing Encode and Conventional Impression Protocols. Int J Oral Maxillofac Implants. 2017;doi: 10.11607/jomi.5386.

[7] Vafiadis DC. Computer-generated abutments using a coded healing abutment: a two year preliminary report. Pract Proced Aesthet Dent. 2007;19(7):443-448.

[8] Ramsey CD, Ritter RG. Utilization of digital technologies for fabrication of definitive implant-supported restorations. J Esthet Restor Dent. 2012;24(5):299-308.

[9] Priest G. Virtual-designed and computer-milled implant abutments. J Oral Maxillofac Surg. 2005;63(9):22-32. 
[10] Mahn DH, Prestipino T. CAD/CAM implant abutments using coded healing abutments: a detailed description of the restorative process. Compend Contin Educ Dent. 2013;34(8):612-615.

[11] Schepke U, Meijer HJA, Kerdijk W, Raghoebar GM, Cune M. Stock Versus CAD/CAM Customized Zirconia Implant Abutments - Clinical and Patient-Based Outcomes in a Randomized Controlled Clinical Trial. Clin Implant Dent Relat Res. 2017;19(1):74-84.

[12] Sailer I, Zembic A, Jung RE, Siegenthaler D, Holderegger C, Hammerle CH. Randomized controlled clinical trial of customized zirconia and titanium implant abutments for canine and posterior single-tooth implant reconstructions: preliminary results at 1 year of function. Clin Oral Implants Res. 2009;20(3):219-225.

[13] Altman DG, Schulz KF, Moher D, et al. The revised CONSORT statement for reporting randomized trials: explanation and elaboration. Ann Intern Med. 2001;134(8):663-694.

[14] Gervais MJ, Hatzipanagiotis P, Wilson PR. Cross-pinning: the philosophy of retrievability applied practically to fixed, implant-supported prostheses. Aust Dent J. 2008;53(1):74-82.

[15] Carrillo de Albornoz A, Vignoletti F, Ferrantino L, Cardenas E, De Sanctis M, Sanz M. A randomized trial on the aesthetic outcomes of implant-supported restorations with zirconia or titanium abutments. J Clin Periodontol. 2014;41(12):1161-1169.

[16] Zembic A, Bosch A, Jung RE, Hammerle $\mathrm{CH}$, Sailer I. Five-year results of a randomized controlled clinical trial comparing zirconia and titanium abutments supporting single-implant crowns in canine and posterior regions. Clin Oral Implants Res. 2013;24(4):384-390.

[17] Albrektsson T, Zarb G, Worthington P, Eriksson AR. The long-term efficacy of currently used dental implants: a review and proposed criteria of success. Int J Oral Maxillofac Implants. 1986;1(1):11-25.

[18] Papaspyridakos P, Chen CJ, Singh M, Weber HP, Gallucci GO. Success criteria in implant dentistry: a systematic review. J Dent Res. 2012;91(3):242-248. 
[19] Gothberg C, Andre U, Grondahl K, Thomsen P, Slotte C. Bone Response and Soft Tissue Changes Around Implants With/Without Abutments Supporting Fixed Partial Dentures: Results From a 3-Year, Prospective, Randomized, Controlled Study. Clin Implant Dent Relat Res. 2016;18(2):309-322.

[20] Abduo J, Lyons K. Rationale for the use of CAD/CAM technology in implant prosthodontics. Int J Dent. 2013;doi: 10.1155/2013/768121.

[21] Lops D, Bressan E, Parpaiola A, Sbricoli L, Cecchinato D, Romeo E. Soft tissues stability of cad-cam and stock abutments in anterior regions: 2-year prospective multicentric cohort study. Clin Oral Implants Res. 2015;26(12):1436-1442.

[22] Zarone F, Sorrentino R, Vaccro F, Russo S, De Simone G. Retrospective Clinical Evaluation of 86 Procera AllCeram Anterior Single Crowns on Natural and ImplantSupported Abutments. Clin Implant Dent Relat Res. 2005;7(1):95-103.

[23] Zembic A, Philipp AO, Hämmerle CH, Wohlwend A, Sailer I. Eleven-Year Follow-Up of a Prospective Study of Zirconia Implant Abutments Supporting Single All-Ceramic Crowns in Anterior and Premolar Regions. Clin Implant Dent Relat Res. $2015 ; 17(2): 417-426$.

[24] Zembic A, Kim S, Zwahlen M, Kelly JR. Systematic review of the survival rate and incidence of biologic, technical, and esthetic complications of single implant abutments supporting fixed prostheses. Int J Oral Maxillofac Implants. 2014;29(Suppl):99-116.

[25] Kapos T, Evans C. CAD/CAM technology for implant abutments, crowns, and superstructures. Int J Oral Maxillofac Implants. 2014;29(Suppl):117-136.

[26] Ferrari M, Tricarico MG, Cagidiaco MC, et al. 3-Year Randomized Controlled Prospective Clinical Trial on Different CAD-CAM Implant Abutments. Clin Implant Dent Relat Res. 2016;18(6):1134-1141.

[27] Worni A, Kolgeci L, Rentsch-Kollar A, Katsoulis J, Mericske-Stern R. Zirconia-Based Screw-Retained Prostheses Supported by Implants: A Retrospective Study on Technical Complications and Failures. Clin Implant Dent Relat Res. 2015;17(6):10731081. 
[28] Krennmair G, Schmidinger S, Waldenberger O. Single-tooth replacement with the Frialit-2 system: a retrospective clinical analysis of 146 implants. Int J Oral Maxillofac Implants. 2002;17(1):78-85.

[29] Lee JH, Lee JB, Kim MY, Yoon JH, Choi SH, Kim YT. Mechanical and biological complication rates of the modified lateral-screw-retained implant prosthesis in the posterior region: an alternative to the conventional Implant prosthetic system. $J$ Adv Prosthodont. 2016;8(2):150-157.

[30] Sambrook RJ, Judge RB, Abuzaar MA. Strategies for restoration of single implants and use of cross-pin retained restorations by Australian prosthodontists. Aust Dent J. 2012;57(4):409-414.

[31] Greenstein G, Carpentieri J, Cavallaro J. Open contacts adjacent to dental implant restorations: Etiology, incidence, consequences, and correction. J Am Dent Assoc. 2016;147(1):28-34.

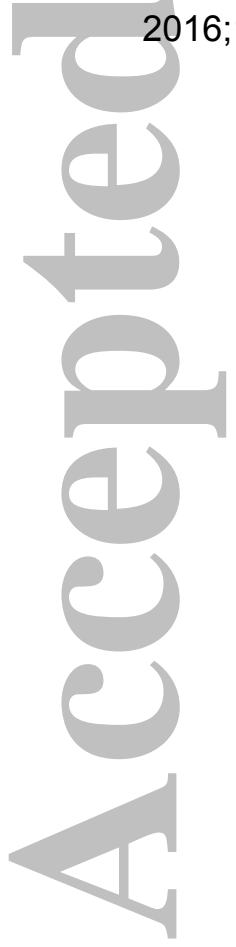


Figure legend

FIGURE 1 The CONSORT flow diagram

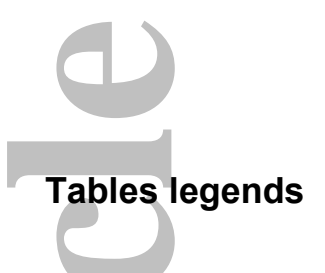

TABLE 1 Mean and standard deviation (SD) of probing pocket depth (PPD) around all

implants, implants restored according to Encode protocol and implants restored according to conventional protocol

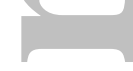

TABLE 2 Proximal contacts quality and occlusal contact quality of the Encode and conventional crowns at baseline and after 1 year

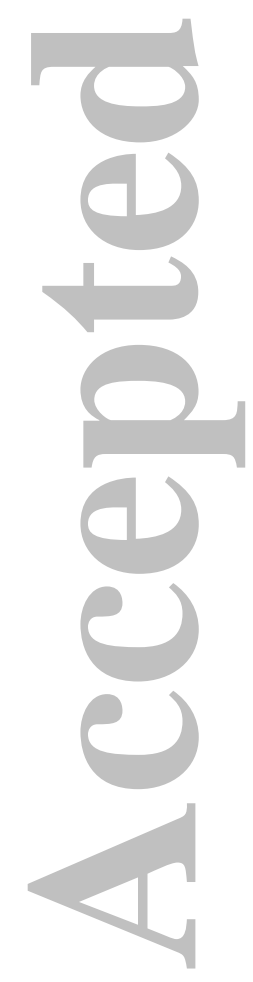


Assessed for eligibility (66 patients)

\section{2 patients excluded}

- 2 patients did not meet the inclusion criteria

- 6 patients refused to participate

- 1 patient was not contactable

- 13 patients required alternative implant system

\section{Enrolment}

44 patients (54 implants)

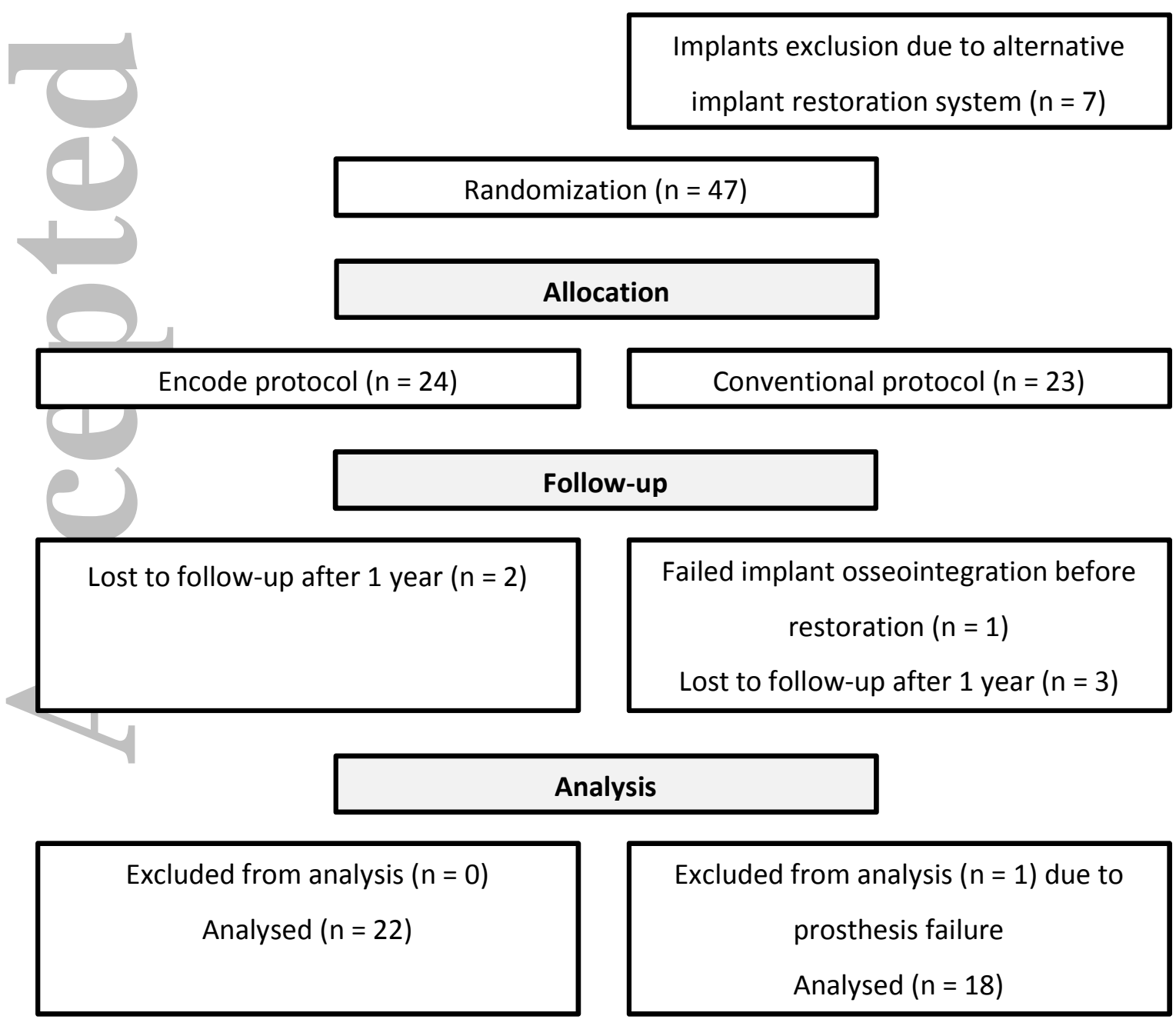


TABLE 1 Mean and standard deviation (SD) of probing pocket depth (PPD) around all implants, implants restored according to Encode protocol and implants restored according to conventional protocol

\begin{tabular}{llll} 
& & Mean $(\mathrm{mm})$ & SD $(\mathrm{mm})$ \\
\hline \multirow{2}{*}{ Baseline } & All & 2.25 & 1.04 \\
& Encode & 2.43 & 1.02 \\
& Conventional & 2.06 & 1.02 \\
Differences after 1 year & All & 2.29 & 1.06 \\
& Encode & 2.41 & 1.08 \\
& Conventional & 2.15 & 1.02 \\
& All & 0.08 & 1.41 \\
& Encode & 0.04 & 1.49 \\
\hline
\end{tabular}

This article is protected by copyright. All rights reserved. 
TABLE 2 Proximal contacts quality and occlusal contact quality of the Encode and conventional crowns at baseline and after 1 year

\begin{tabular}{|c|c|c|c|}
\hline & \multicolumn{3}{|c|}{ Proximal contacts (mesial and distal) } \\
\hline & Quality & Baseline & 1 year \\
\hline \multirow[t]{5}{*}{ Encode } & Open & $3(6.5 \%)$ & $8(18.2 \%)$ \\
\hline & Ideal & $30(65.2 \%)$ & $34(77.3 \%)$ \\
\hline & Tight & $11(23.9 \%)$ & $0(0.0 \%)$ \\
\hline & No adjacent tooth & $2(4.3 \%)$ & $2(4.5 \%)$ \\
\hline & Total & 46 & 44 \\
\hline \multirow[t]{7}{*}{ Conventional } & Open & $3(6.8 \%)$ & $5(13.9 \%)$ \\
\hline & Ideal & $31(70.5 \%)$ & $30(83.3 \%)$ \\
\hline & Tight & $9(20.5 \%)$ & $0(0.0 \%)$ \\
\hline & No adjacent tooth & $1(2.3 \%)$ & $1(2.8 \%)$ \\
\hline & Total & 44 & 36 \\
\hline & \multicolumn{3}{|l|}{ Occlusal contacts } \\
\hline & Quality & Baseline & 1 year \\
\hline \multirow[t]{5}{*}{ Encode } & No adjustment & $1(4.3 \%)$ & $19(86.4 \%)$ \\
\hline & Mild adjustment & $22(95.7 \%)$ & $2(9.1 \%)$ \\
\hline & Major adjustment & $0(0.0 \%)$ & $0(0.0 \%)$ \\
\hline & Out of occlusion & $0(0.0 \%)$ & $1(4.5 \%)$ \\
\hline & Total & 23 & 22 \\
\hline \multirow[t]{5}{*}{ Conventional } & No adjustment & $5(22.7 \%)$ & $17(94.4 \%)$ \\
\hline & Mild adjustment & $15(68.3 \%)$ & $1(5.6 \%)$ \\
\hline & Major adjustment & $1(4.5)$ & $0(0.0 \%)$ \\
\hline & Out of occlusion & $1(4.5 \%)$ & $0(0.0 \%)$ \\
\hline & Total & 22 & 18 \\
\hline
\end{tabular}




\section{University Library}

\section{- M I N E R VA}

\section{A gateway to Melbourne's research publications}

Minerva Access is the Institutional Repository of The University of Melbourne

Author/s:

Abduo, J;Gade, L;Gill, H;Judge, R;Darby, I

Title:

A comparative study of encode protocol versus conventional protocol for restoring single implants: One-year prospective randomized controlled clinical trial

\section{Date:}

2017-12-01

\section{Citation:}

Abduo, J., Gade, L., Gill, H., Judge, R. \& Darby, I. (2017). A comparative study of encode protocol versus conventional protocol for restoring single implants: One-year prospective randomized controlled clinical trial. CLINICAL IMPLANT DENTISTRY AND RELATED RESEARCH, 19 (6), pp.1061-1067. https://doi.org/10.1111/cid.12541.

Persistent Link:

http://hdl.handle.net/11343/293489 